\title{
Spatial Distribution of Pd, Ag \& U in the SiC Layer of an Irradiated TRISO Fuel Particle
}

\author{
Thomas M. Lillo and Isabella J. van Rooyen \\ Idaho National Laboratory, Idaho Falls, ID, USA
}

Advanced nuclear reactor fuel types incorporate various strategies to contain radioactive elements. In the case of tristructural isotropic (TRISO) fuel, a small kernel of enriched uranium carbide oxide (UCO) is encapsulated by a buffer layer of porous carbon, then a layer of pyrolytic carbon (called the inner pyrolytical carbon, IPyC, layer), a silicon carbide ( $\mathrm{SiC}$ ) layer and, finally, an outer pyrolytic carbon (OPyC) layer. The SiC layer performs as a containment barrier to the migration of fission products and eventual release to the reactor coolant. Evidence of the release of Ag through intact TRISO particles has been seen for decades around the world, including the recent AGR-1 experiment at Idaho National Laboratory (INL) [1,2]. Although relatively short lived, this fission product is capable of moving to cooler locations within the reactor system, posing an irradiation hazard during reactor maintenance. Therefore, a full understanding of the migration mechanism of Ag (and other elements) through the $\mathrm{SiC}$ layer is imperative for the further development and licensing of this type of fuel. Silver migration in irradiated $\mathrm{SiC}$ has been the topic of extensive research over the past forty years and many theories have been developed, from microcracks to grain boundary diffusion, all supported by out-of-pile test results, (for a recent review of past data and models, see [3]). However, diffusion coefficients for Ag reported in these works span many orders of magnitude - most likely due to inadequately simulating the $\mathrm{SiC}$ layer of TRISO fuel particles and/or the expected operating conditions - especially irradiation defect generation - in out-of-pile tests. Therefore, this work seeks to determine the spatial distribution of three elements shown to be prevalent - Ag, Pd and U - in TRISO fuel particles exposed to expected, in-pile operating conditions, with the ultimate goal of determining their diffusion coefficients and identifying the underlying mechanism(s) of their movement through the $\mathrm{SiC}$ layer [4].

Sample preparation, equipment limitations and data collection present serious challenges to the characterization of the SiC layer in irradiated TRISO fuel (see Table 1 for irradiation parameters) aside from the requirements needed to handle radioactive materials. In this work, the TRISO particle was embedded in epoxy and polished approximately to the mid-plane of the particle where samples were extracted by standard FIB techniques to produce TEM lamellae. Measuring distances from a feature to the SiC/IPyC interface on these TEM lamellae include errors when the plane-of-polish deviates significantly from the particle mid-plane, Fig. 1a. However, even at deviations of up to $25 \%$ from particle mid-plane ( $\mathrm{x}=0.25$ in Fig. 1a, about $90 \mu \mathrm{m}$ in this work) the distances measured from the IPyC/SiC interface on the TEM sample, d', deviate less than $5 \%$ from the actual distance, $\mathbf{d}_{\mathrm{A}}$. Additionally, the original FIB sample orientation with respect to the radial direction, $\sim 22^{\circ}$ in Fig. $1 \mathrm{~b}$ for samples in this study, must be accounted for when measuring diffusion distances.

Energy dispersive $\mathrm{x}$-ray spectroscopy (EDS) was used to determine the specific elements in features identified in z-contrast images from a high angle annular dark field (HAADF) detector (Tecnai TF30FEG STwin STEM operating at $300 \mathrm{kV}$ at the Center for Advanced Energy Studies, Idaho Falls, ID). In EDS, considerable overlap of the characteristic M-lines for U with the L-lines of both Pd and $\mathrm{Ag}$ exist. So, the characteristic L-lines for $\mathrm{U}$ and the K-lines for Ag and Pd were used to identify these elements in features in lamellae produced at inner (IE), central (Ctr) and outer (OE) locations in the $\mathrm{SiC}$ layer, see Fig. 1b. The features of interest in these samples - grain boundaries, triple junctions and 


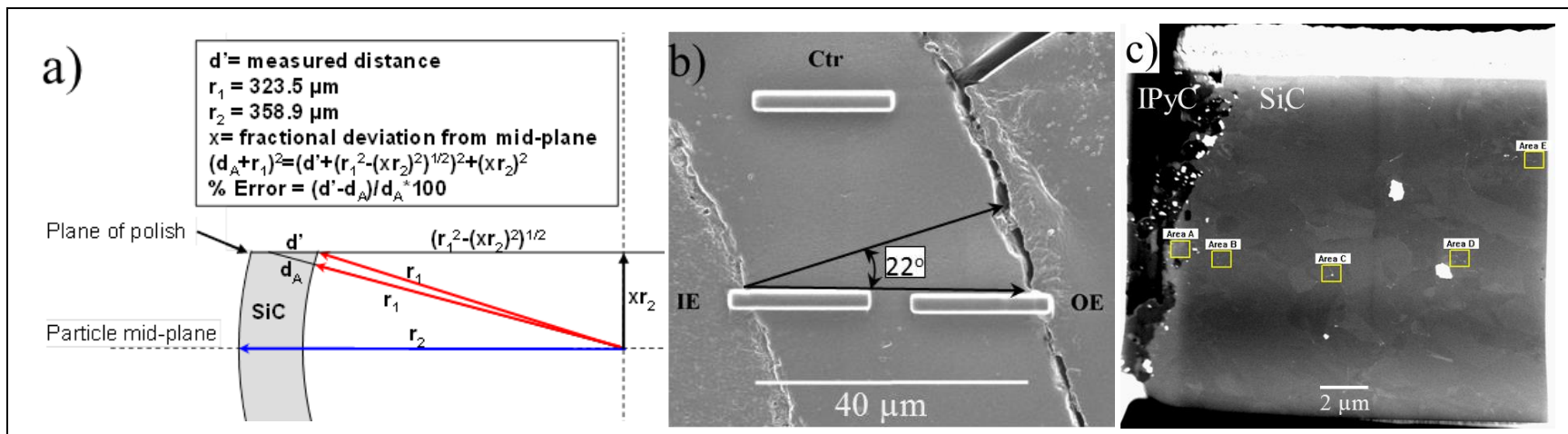

Figure 1. A) Relationship between deviations from mid-plane and errors in distance measured in this side view of a polished TRISO particle , b) top view of location/orientation of three FIB-prepared TEM samples and c) HAADF image of the resulting TEM sample IE in b), showing analysis areas (boxes).

precipitates - are extremely small. Even with an electron beam below $1 \mathrm{~nm}$ in diameter, afforded by the FEG, the surrounding SiC matrix contributed significantly to the EDS spectrum and only the presence

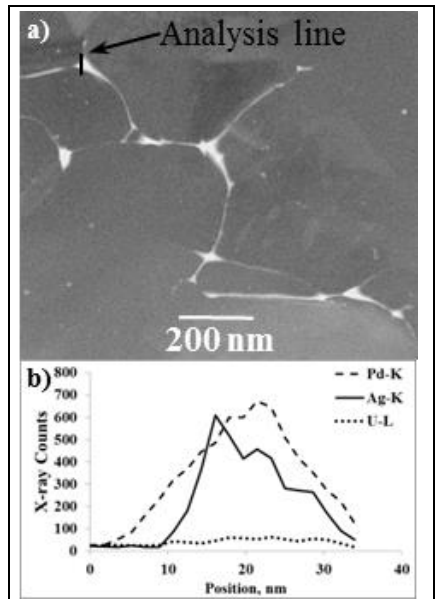

Figure 2. A) Example of EDS analysis line on a triple point and $b$ ) the resulting line profile showing the presence of Ag and $\mathrm{Pd}$.

of an element could be verified. A drift-corrected linescan was set up across a feature of interest, e.g. Fig. 2a, in an analysis area (labeled boxes in Fig. 1c). EDS data was collected for 20 seconds at each point, $\sim 1 \mathrm{~nm}$ increments, in the linescan to produce a composition profile of $x$-ray counts similar to Fig. $2 b$. An element was considered to be present when the position with the maximum number of counts exceeded $50 \%$ of its background value. An extensive number of analyses on the three samples indicated that $\mathrm{Pd}, \mathrm{Ag}$ and $\mathrm{U}$ resided on or at the grain boundaries and triple junctions with only $\mathrm{Pd}$ found in nanoprecipitates in the interior of the $\mathrm{SiC}$ grains. Calculated maximum distances, perpendicular to the $\mathrm{SiC} / \mathrm{IPyC}$ interface, at which $\mathrm{Pd}, \mathrm{Ag}$ and $\mathrm{U}$ were detected, used measurements on STEM images and the angular relationship shown in Fig. 1b. Analysis of the three samples in the Fig.1b revealed that Pd traversed the entire thickness of the $\mathrm{SiC}$ layer while $\mathrm{Ag}$ and $\mathrm{U}$ were detected only about $25 \mu \mathrm{m}$ into the $35 \mu \mathrm{m}$-thick SiC layer. Similar analyses on the SiC layer in other TRISO particles, subjected to irradiation experiments with different parameters will allow calculation of approximate diffusion coefficients to compare with various theories describing $\mathrm{Pd}$, $\mathrm{Ag}$ and $\mathrm{U}$ movement through irradiated $\mathrm{SiC}$.

\section{References}

\section{Table 1. Irradiation Parameters for TRISO Particle}

\begin{tabular}{|l|c|}
\hline \multicolumn{1}{|c|}{ Parameter } & Value \\
\hline$\%$ FIMA average burnup & 11.3 \\
\hline Time-average, volume average temperature, ${ }^{\circ} \mathrm{C}$ & 1070 \\
\hline Time-average, maximum temperature, ${ }^{\circ} \mathrm{C}$ & 1144 \\
\hline Approximate time-at-temperature, days & 620.2 \\
\hline Approximate fast fluence, $\mathrm{n} / \mathrm{cm}^{2}$ & $2.38 \times 10^{23}$ \\
\hline Fuel type & Baseline \\
\hline
\end{tabular}

[1] H. Nabielek, P.E. Brown, P. Offerman, Nucl. Techn. 35 (1977) 483

[2] P.A. Demkowicz, et.al., Paper HTR2012-3-021, Proc. of the HTR 2012, Tokyo, Japan, Oct. 28 Nov. 1, 2012.

[3] J. Malherbe, J. Phys. D: Appl. Phys., 46 (2013) 473001

[4] I.J. van Rooyen, T.M. Lillo, Y.Q. Wu, J. Nuc. Matls, 446 (2014), p.178.

[5]This work sponsored by U.S. DOE, NE, under DOE Idaho Operations Office Contract DE-AC07-

05ID14517 and the authors acknowledge the FIB sample preparation by Jim Madden of the Materials \& Fuels Complex at the INL, the support of the entire MaCS staff at CAES and Scott Ploger for insights on sample preparation . 